Supporting Information

\title{
Mesoscale Ordering of Phycocyanin Molecules in
}

\section{Langmuir-Blodgett Multilayers.}

Eugenia Pechkoval, ${ }^{1,2}$ Claudio Nicolini ${ }^{2}$, Stefano Fiordoro ${ }^{I}$ and Christian Riekeß ${ }^{3}$

${ }^{1}$ Laboratories of Biophysics and Nanotechnology, Department of Experimental Medicine,

University of Genova, Via A. Pastore, 3, 16132 Genova, Italy.

${ }^{2}$ Fondazione EL.B.A - Nicolini, Pradalunga, Bergamo, Italy.

${ }^{3}$ The European Synchrotron, ESRF, CS40220, F-38043 Grenoble Cedex 9, France.

*Corresponding author's e-mail address: eugenia.pechkova@gmail.com 


\section{Materials and Methods}

Protein sample preparation. Native phycocyanin (PC) protein was isolated from cyanobacteria Thermosynechococcus elongatus (T. elongatus). Thermosynechococcus elongatus (T. elongates) cells were preprocessed with a microfluidizer to break the cell walls, followed by a series of centrifugation cycles to isolate the thylakoid membrane. Phycobiliproteins such as PC and allophycocyanin were isolated by ultracentrifugation of the supernatant obtained after microfluidizer treatment. Cell debris and larger particles were spun down at $50000 \mathrm{~g}$ for $1 \mathrm{~h}$. The supernatant was concentrated using Centricon ${ }^{\circledR}$ spin filters with a molecular-weight cutoff of 100 $\mathrm{kDa}$ to obtain protein solution concentrated to $50 \mathrm{mg} \mathrm{ml}^{-1}$.

LB MLs deposition. PC LB MLs were prepared at room temperature (r.t.) by a Nima 611 LB trough using the horizontal lift Langmuir-Schaefer (LS) method. The trough was equipped with a Teflon bath of surface area $300 \times 200 \mathrm{~mm}^{2}$ and $63 \times 180 \mathrm{~mm}^{2}, 70 \mathrm{~mm}$ deep, a Teflon barrier and pressure sensor using a Wilhelmy plate (chromatography paper of wet perimeter $20.6 \mathrm{~mm}$ weight $80 \mathrm{mg} / \mathrm{m}^{2}$ ). The $50 \mathrm{mg} / \mathrm{ml} \mathrm{PC}$ solution in $0.05 \mathrm{M}$ MES buffer with $20 \mathrm{mM} \mathrm{MgCl}_{2}, \mathrm{pH} 6.5$ was spread onto the subphase surface (distilled MilliQ water) by a Hamilton syringe without use of any dispersant. The $200 \mu \mathrm{l}$ of the protein solution of was used for the monolayer formation. Optimal conditions for ordered and well-packed film formation in terms of barriers' speed $\left(100 \mathrm{~cm}^{2} / \mathrm{min}\right)$ and surface pressure of deposition $(26 \mathrm{mN} / \mathrm{m})$ were previously identified ${ }^{2}$. The PC LB $\pi$-A isotherm is shown in the Fig. S1. As the PC is highly soluble, the surface area instead of 
area/molecule was used for the $\pi-\mathrm{A}$ isotherm. The surface pressure chosen for deposition correspond to the close-packing system, as confirmed by QCM. The solid substrates (quartz, mica slide, cryo-EM grid, $\mathrm{Si}_{3} \mathrm{~N}_{4}$ membrane) were firmly kept by the forceps or vacuum gadget (Hampton research, California, USA, HR8-098) for the multiple manual LS deposition. The deposition speed was about $1 \mathrm{~s}$. The transfer ratio of deposition was revealed as close to one.

Every deposited monolayer was dehydrated by nitrogen flux prior to next deposition. The quality of the LB film was controlled by Quartz Crystal Microbalance (QCM) and Atomic Force Microscopy (AFM). 


\section{Surface pressure $\pi, \mathrm{mN} / \mathrm{m}$}

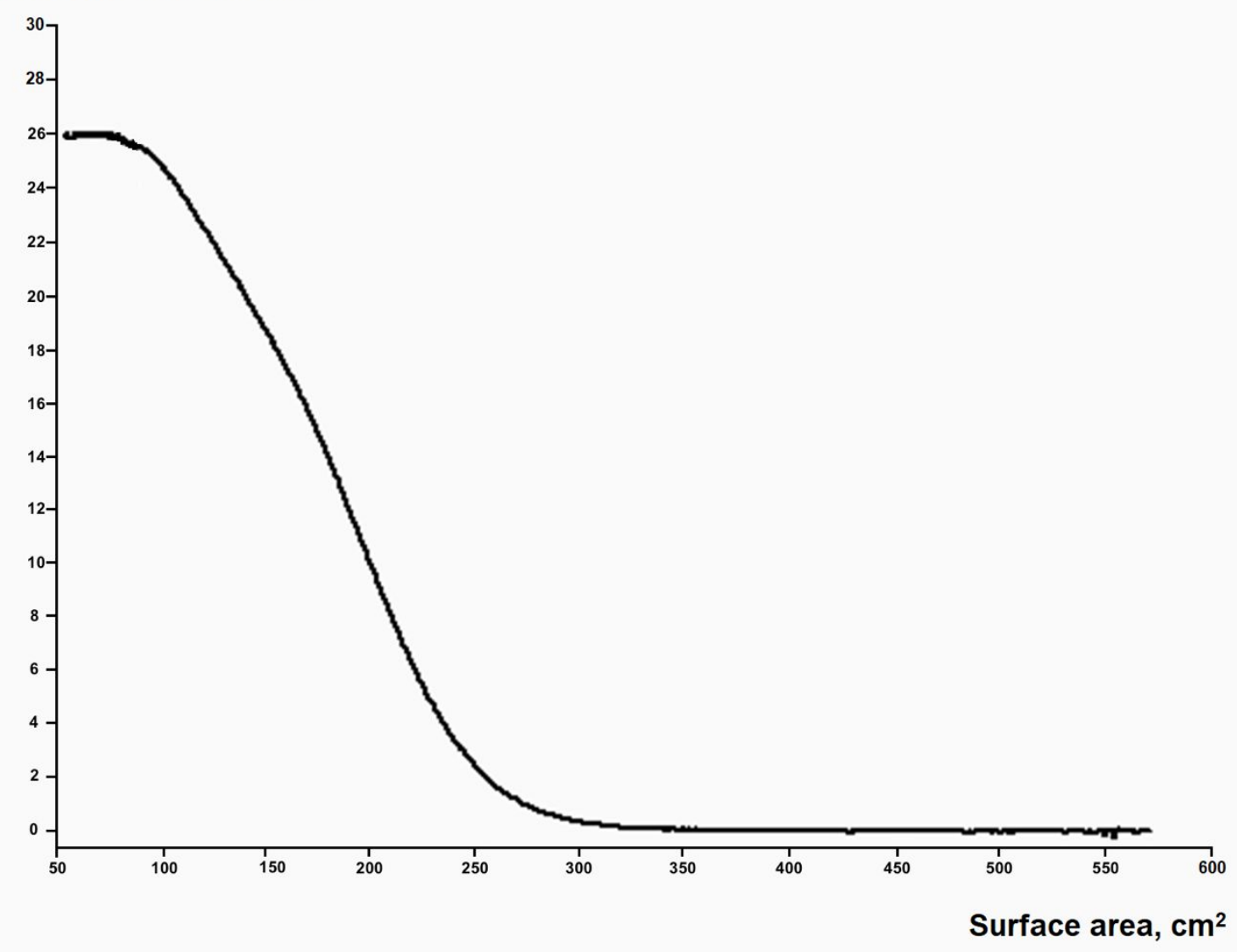

Figure S1. PC LB isotherm at r.t. The surface pressure of PC LB MLs deposition was $26 \mathrm{mN} / \mathrm{m}$.

QCM LB-MLs characterization. QCM exploits the properties of piezoelectric quartz crystals to vary their resonance frequency when a mass is adsorbed to or desorbed from their surface ${ }^{4}$. A QCMagic microbalance by Elbatech, Italy, working at 5-6 MHz was used to characterize the quality (close-packing) of the deposited LB-MLs. We used standard nanogravimetry AT-cut quartz slides of $9.5 \mathrm{MHz}, 14 \mathrm{~mm}$ diameter with $7 \mathrm{~mm}$ gold electrode diameter, 330 microns thickness and 2, HC6/U holder, produced by ICM, Oklahoma City, OK, USA. The quartz slide could be easily mounted and removed from its holding spring contacts for their surface modifications; in our case for the nanofilms deposition on its surface. The frequency shift was 
measured after each LB layer deposition, dried in the gaseous nitrogen flux. Frequency resolution was $\pm 0.05 \mathrm{~Hz}$, quality factor related voltage precision is $0.8 \mathrm{mV}$ and the data acquisition rate is 10 samples/s. The QCMagic software was used for data storage and analysis.

QCM allows correlating the QCM frequency shift $(\Delta f)$ relative to the quartz frequency $\left(f_{0}\right)$ with the mass deposited onto the quartz oscillator $(\Delta m)$ according to the Sauerbrey equation ${ }^{3}$ :

$\frac{\Delta f}{f_{0}}=\frac{\Delta m}{a \rho l}$ S1

or in terms of a surface mass density $\Delta s$ :

$\Delta s=-K \Delta f$ S2

$\left(a\left[\mathrm{~mm}^{2}\right]\right.$ : area covered by protein layer; $\rho\left[\mathrm{g} / \mathrm{mm}^{3}\right]$ : quartz density; $l[\mathrm{~mm}]$ : quartz thickness; $\mathrm{K}$ is a constant related to the impedance of the quartz crystal).

From the experimental data, the frequency shift for each PC monolayer corresponds to a surface mass density of $3 \mathrm{ng} / \mathrm{mm}^{2}$ (Fig. S2). Knowing PC's molecular weight (120 kDa), the experimental surface density value can be used for estimating the number of protein molecules for $1 \mathrm{~mm}^{2}(N)$ and, consequently, the area for one molecule, $A\left[\mathrm{~nm}^{2}\right]$ in the LB monolayer is equal to $67 \mathrm{~nm}^{2}$ :

$N=\left(\Delta s \times N_{A}\right) / M W \times 10^{12}$

$A=10^{12} / N$

Taking into consideration the geometric features of PC protein molecule (PDB code 3L0F, see insert in Fig. S2) one can conclude that the deposited protein monolayers are highly packed and homogeneous and that the deposition of each layer is reproducible. Indeed, based on PC's PDB 
structure, the area of one molecule is determined as about $70 \mathrm{~nm}^{2}$. We conclude therefore that PC molecules pack in the hexameric form in the ML nanofilms.

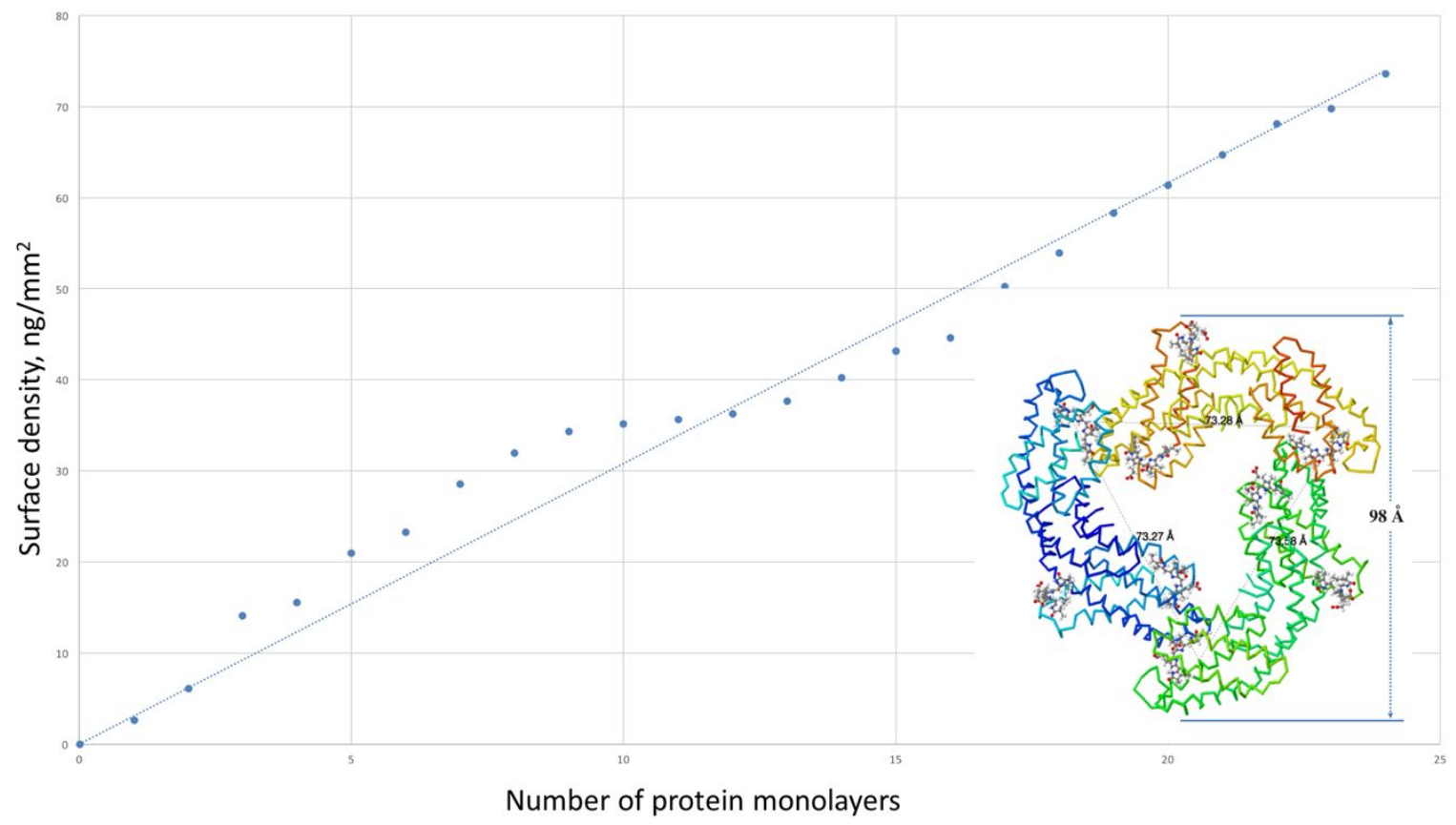

Figure S2. QCM measurement of subsequent 24 PC LB layers deposition onto a quartz oscillator substrate resulting in a linear surface mass density increase with the number of PC monolayers deposited. Each data point corresponds to a deposited monolayer. The inset shows the PC molecule hexamer (PDB: 3L0F; cartoon view provided by WebGL protein viewer ${ }^{4}$ ).

AFM LB-MLs characterization. The AFM was operated in a low thermal drift setting thermal cabinet allowing achieving a better than $0.2 \mathrm{~nm} \mathrm{~min}{ }^{-1}$ drift rate. For low-force imaging we used either a frequency modulation mode with the probe operating at sub-nanometer amplitude or the capabilities of the probe for thermal noise measurements used for surface profiling. PC LB-MLs (20 layers) were deposited on a mica surface by using the LS modification of the LB method, and dried in the nitrogen flux. PC MLs studies were probed in air with a MultiMode microscope and 
Nanoscope IIIA controller (Bruker) as described in ${ }^{5,6}$. AFM was made in tapping mode with a scanner having lateral displacements of up to 150 microns. The scanner was calibrated with 3 micron and $278 \mathrm{~nm}$ gratings. Silicon probes (Applied Nanostructures) with spring constants less than $2 \mathrm{~N} / \mathrm{m}$ were used in this study. In the AFM experiments we applied free probe amplitudes $A_{0}$ $=5-40 \mathrm{~nm}$ and set-point amplitude $\mathrm{A}_{\mathrm{sp}}=0.9-0.5 \mathrm{~A}_{0}$.
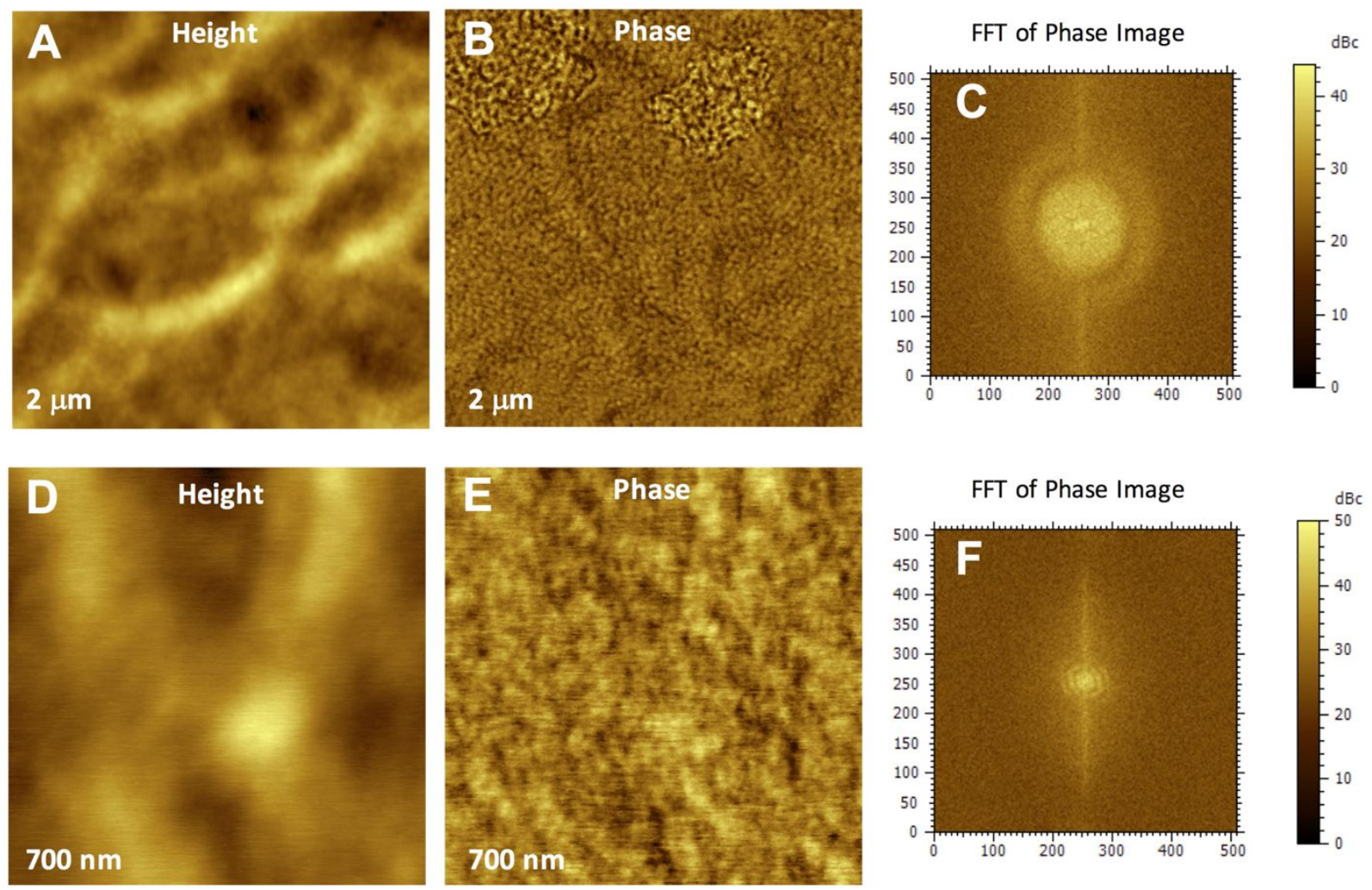

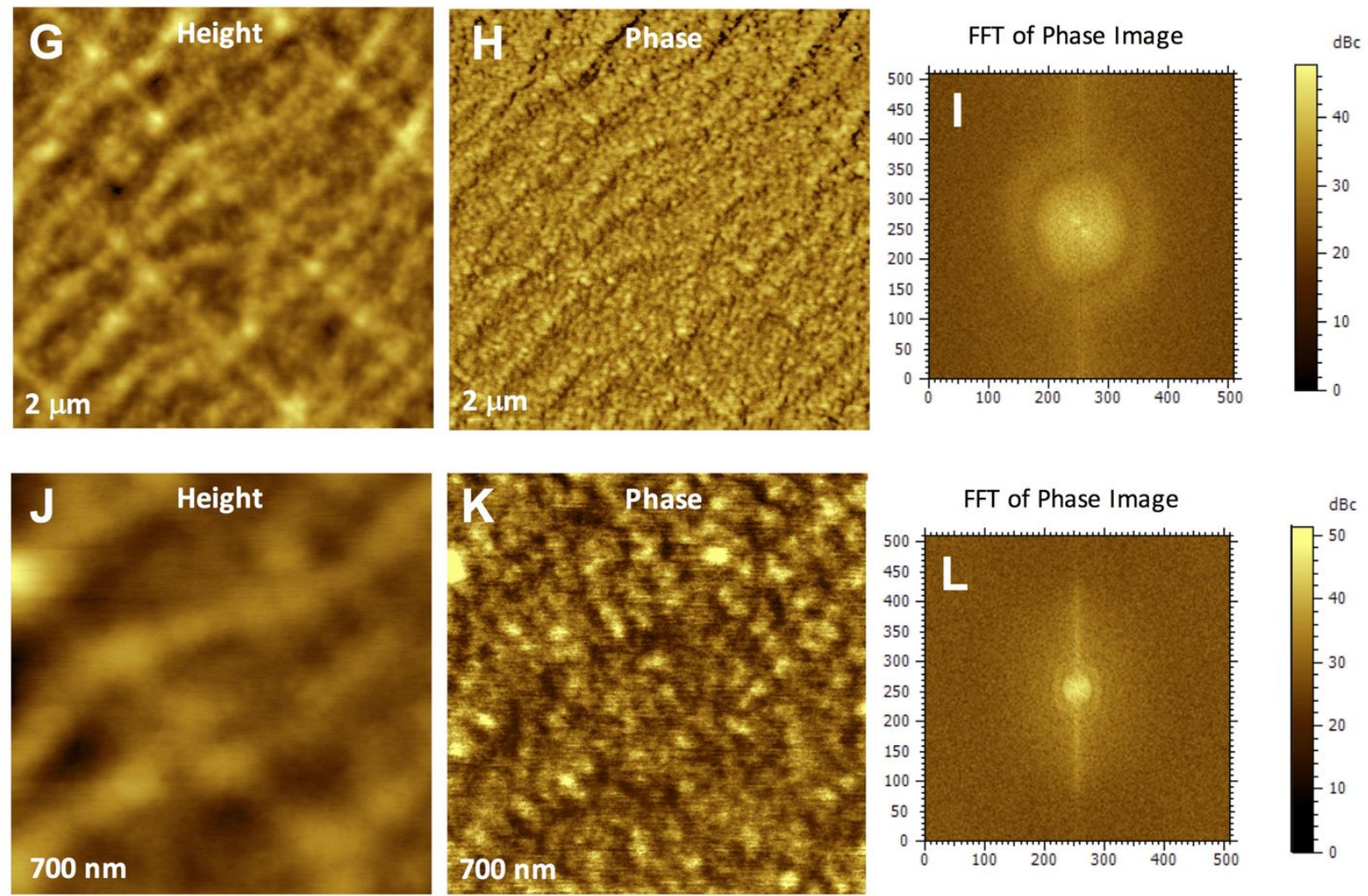

Figure S3. (A-F) Height and phase AFM images of PC LB-MLs (20 layers) at r.t. (before heating), the square measures $2 \times 2$ microns for upper and $700 \times 700 \mathrm{~nm}$ for lower images. (G-L) Height and phase AFM images of PC LB-MLs (20 layers) after heating to $150^{\circ} \mathrm{C}$ for 10 minutes, the square measures $2 \times 2$ microns for upper and 700x700 nm for lower images. The corresponding Fast Fourier Transform (FFT) of phase images are shown on the right.

Scanning rates of $0.6-0.8 \mathrm{~Hz}$ were used for areas of several micron on side, and $1 \mathrm{~Hz}$ rate was applied for sub-micron imaging. Image treatment and analysis was made with MountainsMap software by Digital Surf (France). Height and phase AFM images of PC LB-MLs before and after heating are shown on the Figure S3. 
Cryo-EM grids preparation. Carbon-formvar films were used as a support coating on TEM mesh gold or copper grids. Formvar resin 15/95 (polyvinyl formal, Electron Microscopy Sciences, Cat.\# 15800) was dissolved at $0.5 \%$ concentration overnight in dichloroethane (Electron Microscopy Sciences, Cat.\# 13250) dried by molecular sieves. Films with an approximate thickness of 25-30 $\mathrm{nm}$ were cast by dipping glass microscope slides $(75 \mathrm{~mm} \times 25 \mathrm{~mm})$ into the formvar solution and allowed to air-dry for several minutes. The films were released from the slides onto the surface of deionized water in a large bowl. 300-mesh copper TEM grids were carefully laid onto the floating film in an organized array and eventually picked up on an appropriate substrate. After drying, the formvar supports were coated with a $~ 10$ nm layer of carbon using a Denton Desk IV unit (Denton Vacuum, Moorestown, NJ). TEM grids prepared in this way were used for PC MLs subsequent LS deposition as described above.

MicroED data collection and analysis. Cryo-EM analysis was based on 10-20 LB PC-MLs deposited on EM grids coated by formvar and carbon layers. MicroED was performed in transmission geometry with the beam normal to the sample surface at the John M. Cowley Center for High Resolution Electron Microscopy (CHREM), Arizona State University (USA). We used a Titan Krios EM (FEI) operating a $300 \mathrm{keV}\left(\lambda_{\mathrm{e}}=0.0022 \mathrm{~nm}\right)$, with a spherical aberration (Cs) of 2.7 mm, equipped with a Gatan K2 Summit direct electron detector. ED images were recorded with a 
hybrid CMOS 4K x 4k (Ceta) camera. Cryogenic sample-grids were rapidly transferred and cooled in liquid ethane $\left(-188^{\circ} \mathrm{C}\right)$. Cryo-EM measurements were performed as described by Shi et $\mathrm{al}^{7}$. The diameter of the circular electron beam was estimated from three optical images of the probed samples by an increase of the radiation-induced grey-scale level as $5.6 \pm 0.1 \mu \mathrm{m}$. All patterns contained a $1 \mathrm{~nm}^{-1}$ scale bar. Lattice spacings $(\mathrm{d}$; $\mathrm{nm}$ ) of reflections were obtained from $\mathrm{d}=1 / \mathrm{s}$ where $s\left(\mathrm{~nm}^{-1}\right)$ is the distance of the peak from the origin of reciprocal space. The accuracy of lattice spacing is estimated to be approximately $\pm 0.01 \mathrm{~nm}$. The beamstop was asymmetric, corresponding to a maximum d-spacing at cut-off of $\sim 2 \mathrm{~nm}$.

Scanning nanoXRD was performed at the ID13 beamline of the European Synchrotron (ESRF).

Derived data supporting the findings of this study are available from the corresponding author upon reasonable request. Experiments were performed on previously deposited and thermally annealed PC LB-MLs using a monochromatic beam of $1=0.08157 \mathrm{~nm}$ focused to about $170 \times 170$ $\mathrm{nm}^{2}$ at the sample position 9 . The samples were imaged and positioned in the focal spot by an onaxis Olympus microscope. NanoXRD mesh-scans were performed for two samples in transmission geometry along vertical and horizontal axes, in a plane normal to the beam direction ${ }^{8}$ for a $180 \times 180$ $\mu \mathrm{m}^{2}$ sample area with $1 \mu \mathrm{m}$ step-increments. The samples were mesh-scanned through the beam using a PI M-810 6-Axis Hexapod PI with $\pm 500 \mathrm{~nm}$ repeatability. At each step a $0.2 \mathrm{~s}$ diffraction pattern was recorded by an Eiger 4M pixel detector (DECTRIS) with 2070x2167 pixels of $75 \mu \mathrm{m}$ $\mathrm{x} 75 \mu \mathrm{m}$ area each, single photon counting capability and no readout noise. The sample-to-detector distance was determined by an Ag-behenate powder standard ${ }^{9}$ as $403.1 \mathrm{~mm}$. The beamstop was approximately symmetric, corresponding to a d-spacings at cut-off of $\sim 31 \mathrm{~nm}$. The results of the 
mesh-scans are displayed as composite X-ray density maps using FIT2D ${ }^{10}$ software routines (Figs.

4A,B). 


\section{References:}

1 R. Fromme, A. Ishchenko, M. Metz et al., "Serial femtosecond crystallography of soluble proteins in lipidic cubic phase," IUCrJ, 2, 545-51 (2015).

2 E. Pechkova. C. Nicolini. "Langmuir-Blodgett protein multilayer nanofilms by XFEL," NanoWorld J 4, 48-53 (2018).

3 G. Sauerbrey, "Verwendung von Schwingquarzen zur Waegung duenner Schichten und zur Mikrowaegung," Zeitschrift fuer Physik 155, 206-222 (1959); Y. Jiménez, M. Otero, A. Arnau, in Piezoelectric Transducers and Applications, edited by A. Arnau (Springer, Berlin, 2008), pp. 331-398.

4 A.S. Rose, A.R. Bradley, Y. Valasatava et al., "NGL viewer: web-based molecular graphics for large complexes," Bioinformatics 34, 3755-3758 (2018).

5 A. Koyfman, S. Magonov, N. Reich, Self-assembly of DNA arrays into multilayer sheets, Langmuir 25, 1091-1095 (2009).

6 I. Yermolenko, A. Fuhrmann, S. Magonov et al., "Origin of the non-adhesive properties of fibrinogen matrices probed by force microscopy," Langmuir 26, 17269-1727 (2010).

7 D. Shi, B.L. Nannenga, M.G. Iadanza et al., "Three-Dimensional Electron Crystallography of Protein Microcrystals," eLife 2, 01345 (2013).

8 C. Riekel, M. Burghammer, I. Snigirev et al. "Microstructural metrology of tobacco mosaic virus nanorods during radial compression and heating," Soft Matter 14, 194-204 (2018).

9 T.N. Blanton, T. C. Huang, H. Toraya et al. "JCPDS-International Centre for Diffraction Data round robin study of silver behenate. A possible low-angle X-ray diffraction calibration standard." Powder Diffraction 10, $91-95$ (1995).

10 A. Hammersley (2009). The FIT2D Home Page. www.esrf.fr/computing/scientific/FIT2D/. Grenoble, ESRF. 
